\title{
BUCKLING OF HEAVY BARS
}

By Dr.-Ing. Aydoğan Özdamar

1030 Sok. Ögretmenler Sitesi

A Blok Kat:2 Daire:4

Buca-IZMiR

ABSTRACT: The calculation for the buckling of a short bar under compression occurs normally through the neglect of its own weight. This method is no longer permissible for long heavy bars. In this case, the net weight must be taken into consideration. Such long heavy bars can be found, for example, as drilling-risers in ocean exploration technology, as schafts in mining, as steel turrets for the production of energy and as naturally grown mammoth trees.

In this paper, the critical buckling forces are derived from ten end conditions for heavy bars which are surrounded by air and burdened by compression. There is an analytical solution to the differential equation of the problem which requires a numerical evaluation. Limited computer capacity permits calculations of critical buckling forces only up to a certain length. An analytical solution to the differential equation must be developed asymptotically for long heavy bars. With the help of this asymptotic evaluation, the critical buckling forces for long heavy bars are found.

\section{Introduction}

In general the buckling of a short bar under compression is calculated by ignoring the bar's own weight. This method, however, can not be applied to long bars for some economical reasons.

It is possible to see long bar applications in different industrial areas; such as drilling risers in mining and oil exploration, long chimneys, and steel towers to produce electric energy from wind.

With considering bar's own weight, Greenhill [5] (1881) was the first to calculate critical buckling load under compression for one end condition. Later on Willers [9] (1941) calculated these loads for five different end conditions of limited long bars by using Bessel and Lommel functions. Airy functions were used by Plunkett [8] (1967) to find the buckling loads of very long bars for two end conditions. Hapel [6] (1976) also used Bessel and Lommel functions for his calculations for one end condition, and his result was different than Willers'. In their study, Bernitsas and Kokkinis [1-4] (1981-1984) used Airy functions to calculate buckling forces for eight different end conditions. Their result and Hapel's are similar although they used different methods. They also pointed out that Willers' some results were different because Willers took only limited number of asymptotic seri members into account.

The Author [7] (1996) used Bessel and Lommel functions to calculate buckling loads of heavy bars under compression for ten different end conditions. The results show that Bernitsas and Kokkinis' calculations are correct. However, this paper will show that Willers' fault was not of taking only the first two terms of the asymptotic series into account as Bemitsas and Kokkinis claimed.

Following calculations are taken from Özdamar's [7] (1996) study. 


\section{Differential Equation of Problem and Solution}

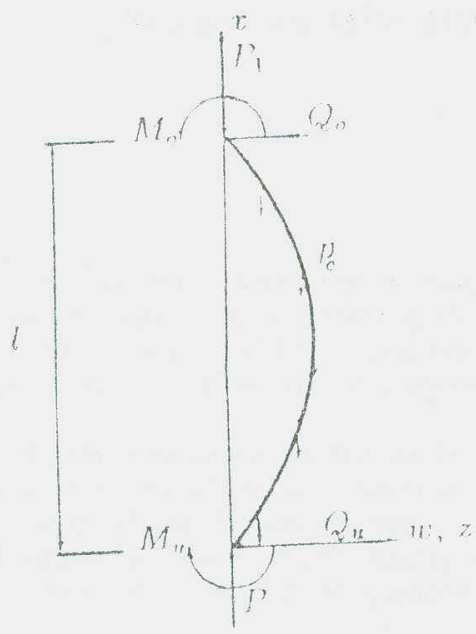

Figure 1: Free body diagram

As can be seen from the free body diagram of a bar, following differential equation is found when calculating static balance forces and moments:

$\frac{d^{3} w(x)}{d x^{3}}+a(b-x) \frac{d w(x)}{d x}=\mathrm{k}$

where

$a=\frac{p_{o}}{E I}>0, b=\frac{P}{p_{0}}>0$

By using

$\zeta(x)=\frac{2}{3} \sqrt{a} \sqrt{(b-x)^{3}} \quad$ and

$\frac{d w(x)}{d x}=[\zeta(x)]^{\frac{1}{3}} \phi(\zeta(x))$

this differential equation converted to the following form:

$\phi^{\prime \prime}(\zeta)+\frac{1}{\zeta} \phi^{\prime}(\zeta)+\left(1-\frac{1}{9 \zeta}\right) \phi(\zeta)=\frac{C_{1}}{\zeta}$. 
This equation is called as Bessel Differential equation. Here,

$$
C_{1}=\mathbf{k}\left(\frac{2}{3 a}\right)^{\frac{2}{3}}
$$

The solution of this differential equation is known:

$$
\phi(\zeta)=C_{1} s_{0, \frac{1}{3}}(\zeta)+C_{2} J_{\frac{1}{3}}(\zeta)+C_{3} J_{-\frac{1}{3}}(\zeta)
$$

In this equation $J_{\frac{1}{3}}(\zeta)$ and $J_{-\frac{1}{3}}(\zeta)$ are called as Bessel functions, and $s_{0, \frac{1}{3}}(\zeta)$ is called as Lommel function.

With the help of this solution $w$ and derivatives of $w$ are calculated from equation (1). From end conditions, the critical buckling forces are calculated for every ten end conditions.

As ( $\zeta$ ) is getting higher, in these Bessel and Lommel functions a great number of series' terms should be taken into account, if computer has sufficient capacity. In this case asymptotic expansions can be use. At the top end of the bar the specific variable of equation, which can be expressed by asymptotic expansions, is as follows:

$\zeta_{x=1}= \pm \eta_{u}(\alpha-1)^{\frac{3}{2}} \mathrm{i}= \pm \eta_{0} \mathrm{i}$,

where

$\eta_{u}=\frac{2}{3} a^{\frac{1}{2}} b^{\frac{3}{2}}, \eta_{o}=\eta_{u},(\alpha-1)^{\frac{3}{2}}, \alpha=\frac{l}{b}$.

Table 1 below shows Bessel and Lommel expansions for the top end of the bar with comparison of Willers' results. From these expansions derivatives of $w$ in equation (1) can be found. From end conditions, critical buckling forces of very long bars can also be

\begin{tabular}{|c|c|c|c|}
\hline & $\zeta=\eta_{0} i$ & $\zeta=-\eta_{a} i$ & $\zeta=\eta_{0}^{i}$ (WILLERS) \\
\hline$J_{\frac{1}{3}}(\zeta) \sim$ & $e^{\left(\frac{\pi}{5}-k \pi\right) i} g(\eta) T_{2}(\eta)$ & $e^{\left(-\frac{\pi}{6}-k \pi\right) i} g(\eta) T_{2}(\eta)$ & $e^{\frac{\pi}{6} i} g(\eta)\left(1-\frac{1}{6 \eta}\right)$ \\
\hline$J_{-\frac{1}{3}}(\zeta) \sim$ & $e^{\left(-\frac{\pi}{6}-k \pi\right) i} g\left(\eta_{\partial} T_{2}(\eta)\right.$ & $e^{\left(\frac{T}{\varepsilon}-k \pi\right) i} g\left(\eta_{d}\right) T_{2}(\eta)$ & $e^{-\frac{\pi}{6} i} g(\eta)\left(1+\frac{1}{6 \eta_{0}}\right)$ \\
\hline$s_{0, \frac{1}{3}}(\zeta) \sim$ & $\frac{1}{i} T_{3}(\eta) \pm \frac{\pi}{i \sqrt{3}} g(\eta) T_{2}(\eta)$ & $\frac{-1}{i} T_{3}\left(\eta_{0}\right)=\frac{\pi}{i \sqrt{3}} g(\eta) T_{2}(\eta)$ & $\frac{-\pi}{i \sqrt{3}} g(\eta)\left(1-\frac{1}{6 \eta}\right)$ \\
\hline
\end{tabular}
calculated. Solutions of ten different end conditions are presented in Figure 2.

Table 1: Asymptotic expansions of Bessel and Lommel functions 
In this table,

$g\left(\eta_{0}\right)=\frac{e^{\eta_{o}}}{\sqrt{2 \pi \eta_{o}}}, \mathrm{k}=0,1$

and, $T_{2}\left(\eta_{0}\right)$ and $T_{3}\left(\eta_{0}\right)$ series are semi-konvergent and formulations are as follows:

$\mathrm{T}_{2}\left(\eta_{0}\right) \sim 1+\frac{5}{72 \eta_{0}}+\ldots, \mathrm{T}_{3}\left(\eta_{0}\right) \sim \frac{1}{\eta_{0}}+\frac{8}{9 \eta_{o}}+\ldots$

As can be considered from Table 1 , Willers did not consider $-\eta_{0} \mathrm{i}$ as $\eta_{0} \mathrm{i}$. Secondly in the Lommel function expansion $T_{3}\left(\eta_{0}\right)$ was ignored by Willers. Finally instead of $T_{2}\left(\eta_{0}\right)$, he found the equations in the brackets which are not correct.

\section{Conclusion}

By using dimensionless buckling force of

$\bar{P}=\frac{P}{\sqrt[3]{E I p_{0}^{2}}}$

and using dimensionless bar length of

$\vec{l}=\sqrt[3]{\frac{p_{o}}{E I}}$

critical buckling forces are calculated for ten different end conditions. Results are shown in Table 2, with comparisons of other authers.

In conclusion, this study solves the conflict between two different methods; Airy and Bessel functions, when calculating critical buckling forces of very long bars.

\section{$\underline{\text { References }}$}

[1] Bernitsas, M.-Kokkinis, T. : Asymptptic Behavior of Riser Stability Boundaries, Publication 255, Department of Naval Architecture and Marine Engineering, The University of Michigan, Ann Arbor, 1982.

[2] Bernitsas, M--Kokkinis, T. : Buckling of Columns with Nonmovable Boundaries, Journal of Structural Engineering 109, 1983, p. 2113-2128.

[3] Bernitsas, M.-Kokkinis, T. : Buckling of Columns with Movable Boundaries, Journal of Structural Mechanics 11, 1983, p. 351-370. 
[4] Bernitsas, M.-Kokkinis, T. : Asymptotic Behavior of Heavy Column and Riser Stability Boundaries, Journal of Applied Mechanics 51, 1984, p. 560-565.

[5] Greenhill, A. G. : On Height Consistent With Stability, Proceedings of the Cambridge Philosophical Society, Vol. IV, 1881, p. 5-7.

[6] Hapel, K. H. : Festigkeitsanlyse dynamisch beanspruchter Offshore-Konstruktionen, Vieweg Verlag, Wiesbaden, 1990.

[7] Özdamar, A. : Das Knicken schwerer Gestaenge, VWF Verlag für Wissenschaft und Forschung, ISBN 3-930324-71-7, Berlin, 1996.

[8] Plunkett, R. : Static Bending Stresses in Catenaries and Drill Strings, Journal of Engineering for Industry 89, 1967, p. 31-36.

[9] Willers, F. A. : Das Knicken schwerer Gestaenge, Z. angew. Math. Mech., Bd. 21, Dresden, 1941, p.43-51.

\begin{tabular}{|c|c|c|c|c|c|c|c|c|c|c|}
\hline \multirow{2}{*}{ Author } & \multicolumn{10}{|c|}{ End Conditions } \\
\hline & II & 12 & 13 & 14 & 21 & 22 & 23 & 31 & 32 & प1 \\
\hline WLLLERS (1941) & 3,09 & 1,88 & - & 1,0188 & 3,09 & 1,88 & - & - & - & - \\
\hline PLUNKETT (1967) & - & - & - & 1,0188 & - & - & - & - & - & 2,3381 \\
\hline HAPEL (1976) & - & - & - & - & - & 1.02 & - & - & - & - \\
\hline BERN.-KOK. (1982) & 2,338 & 1,018 & - & 1,018 & 2,338 & 1,018 & - & 2.338 & 1,018 & 2,3381 \\
\hline nZDAHAR (1996) & 2,3381 & 1,0188 & 2,3381 & 1,0188 & 2,3381 & 1,0188 & 2,3381 & 2.3381 & 1,0188 & 2,3381 \\
\hline
\end{tabular}

Table 2: Comparison of dimensionless critical buckling forces 


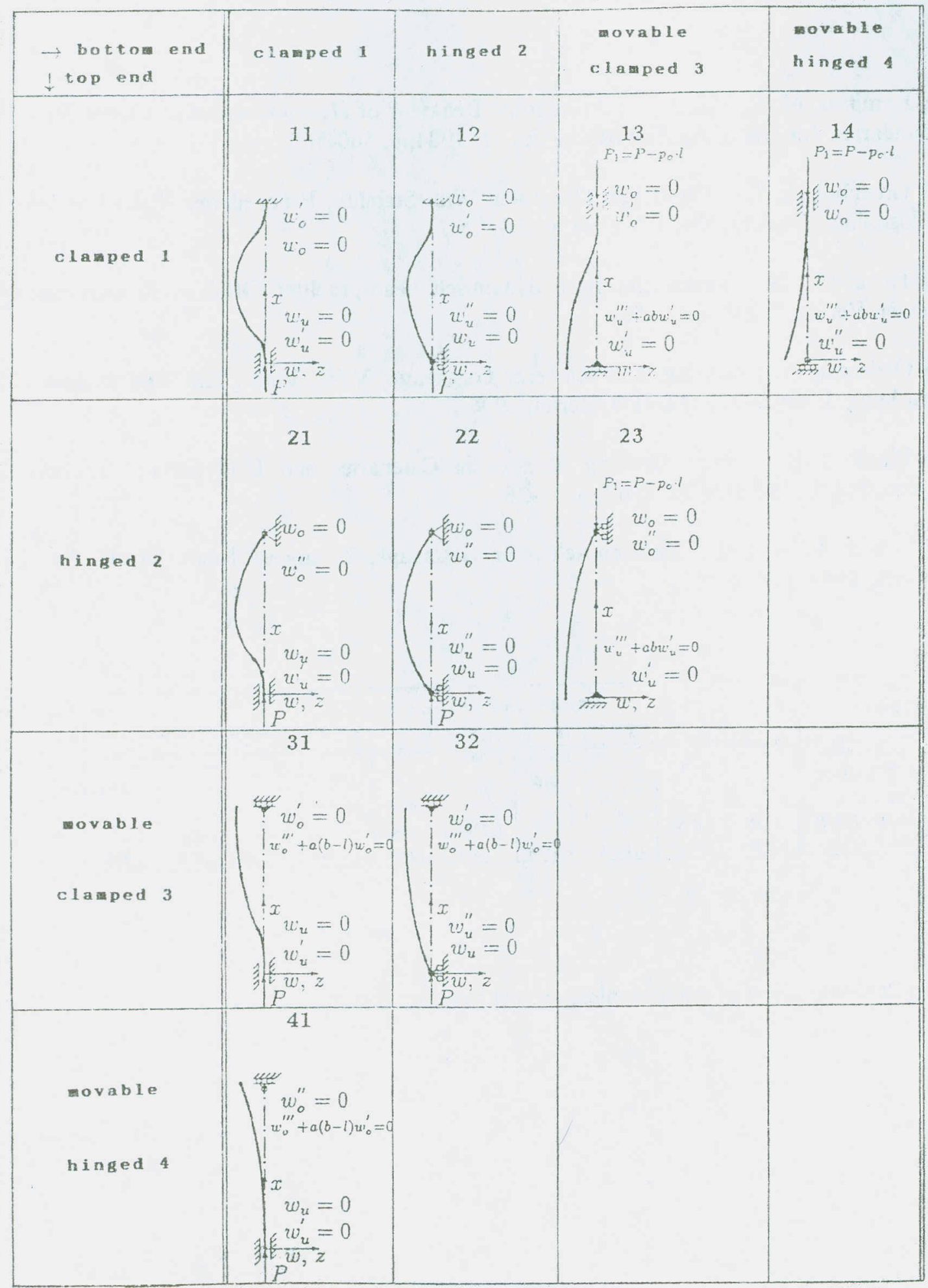

Figure 2: Ten end conditions calculated in this study 


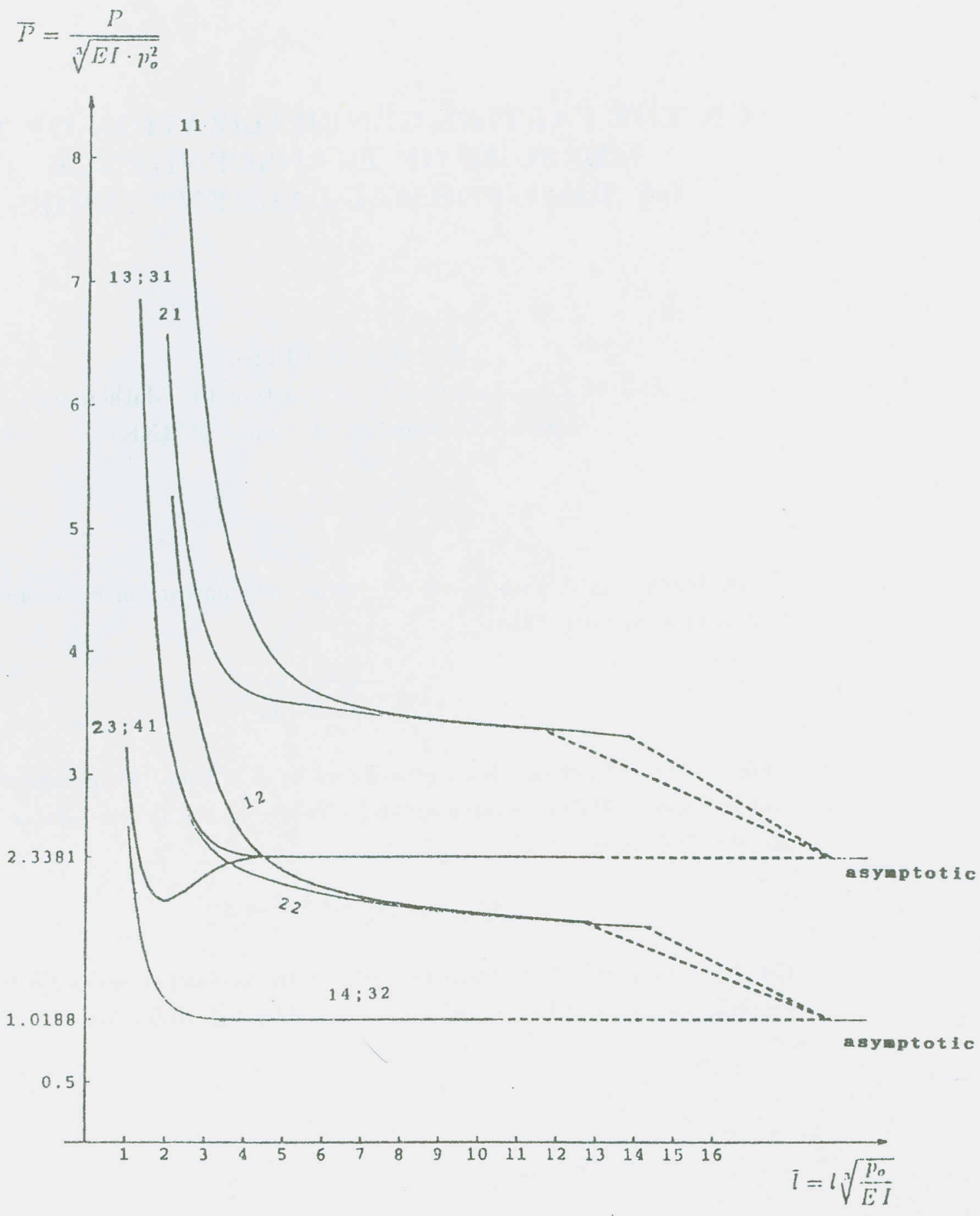

Figure 3: Dimensionless critical buckling forces vs dimensionless bar length 